\title{
Smokers with Multiple Sclerosis Are More Likely to Report Comorbid Autoimmune Diseases
}

\author{
Ruth Ann Marrie $^{a}$ Ralph I. Horwitz ${ }^{b}$ Gary Cutter $^{c}$ Tuula Tyry ${ }^{d}$ \\ Timothy Vollmer ${ }^{\mathrm{d}}$ \\ ${ }^{a}$ Department of Internal Medicine, University of Manitoba, Winnipeg, Man., Canada; ${ }^{b}$ Department of Medicine, \\ Stanford University, Stanford, Calif., 'Department of Biostatistics, University of Alabama at Birmingham, \\ Birmingham, Ala., and ${ }^{\mathrm{d} D i v i s i o n}$ of Neurology, Barrow Neurological Institute, Phoenix, Ariz., USA
}

\section{Key Words}

Smoking $\cdot$ Comorbidity $\cdot$ Autoimmune disease $\cdot$

Multiple sclerosis $\cdot$ Risk factor

\begin{abstract}
Background/Aims: Smoking is a risk factor for multiple sclerosis (MS) and autoimmune disease, and might explain an increased risk of comorbid autoimmune disease (CAD) in MS. We compared the risk of CAD in smokers and nonsmokers with MS. Methods: Participants enrolled in the North American Research Committee on Multiple Sclerosis Registry reported their smoking status, the presence of CAD and the year of diagnosis. We used multivariable logistic regression to determine the independent association between smoking and CAD. We also compared the risk of developing a CAD in current smokers versus never-smokers who did not report any CAD at MS onset, using a proportional hazards model. Results: Among 8,875 participants reporting comorbidities and smoking status, 1,649 (18.5\%) reported a CAD. In a multivariable logistic model, ever-smokers had increased odds of reporting a CAD (odds ratio: 1.22; 95\% Cl: 1.08-1.38). Among the 7,830 participants without a CAD at onset of MS
\end{abstract}

who reported their smoking status, including the age at which they started smoking, 3,035 (36.8\%) currently smoked, while 3,805 (48.6\%) never smoked. After adjustment, smokers had an increased risk of developing any autoimmune disease (hazard ratio: 1.23; 95\% Cl: 1.08-1.41) after MS onset. Conclusion: Smoking is associated with an increased risk of CAD in MS.

Copyright $\odot 2011$ S. Karger AG, Basel

\section{Introduction}

Several studies suggest an association between autoimmune diseases and multiple sclerosis (MS) $[1,2]$. Often, such associations are interpreted to indicate a shared genetic susceptibility, but an alternative explanation would be a shared environmental exposure, such as smoking. Smoking is a reported risk factor for MS [3] and other autoimmune diseases including rheumatoid arthritis, inflammatory bowel disease and, possibly, uveitis $[4,5]$. In MS, it is unknown whether smoking influences the risk of comorbid autoimmune disease (CAD). Further, findings regarding the association of autoimmune diseases

Ruth Ann Marrie, MD, $\mathrm{PhD}$

Health Sciences Center, GF 543

820 Sherbrook Street

Winnipeg, MB R3A 1R9 (Canada)

Tel. +1 204787 4951, Fax +1 204787 1486, E-Mail rmarrie@ hsc.mb.ca 
with MS are inconsistent. If smoking were associated with an increased risk of autoimmune disease in MS, this would be important for designing future studies assessing genetic or environmental associations between MS and autoimmune disease. We investigated whether smokers with MS had an increased risk of CAD versus nonsmokers.

\section{Participants and Methods}

The study methods are detailed elsewhere [6]. Briefly, the North American Research Committee on Multiple Sclerosis (NARCOMS) Registry is a self-report registry for MS. The NARCOMS Registry is approved by the institutional review board at the University of Alabama at Birmingham. Participants give permission for their information to be used for research purposes.

Participants self-report demographic and clinical information at enrollment and semiannually thereafter. In 2006, we queried participants regarding autoimmune comorbidities including rheumatoid arthritis, Sjögren's syndrome, systemic lupus erythematosus, inflammatory bowel disease, autoimmune thyroid disease and uveitis; conditions were selected based on their reported associations with MS, or if they were conditions frequently included in existing comorbidity instruments including self-report questionnaires $[7,8]$. For thyroid disease, participants were asked to report any autoimmune thyroid disease, but they were not asked to distinguish between Graves' disease and Hashimoto's thyroiditis, in the absence of existing literature supporting the ability of participants to distinguish these by self-report. Because this study was part of a larger study examining comorbidities in MS, we did not attempt to capture all possible autoimmune comorbidities to minimize participant response burden. For analysis, we created a single variable, any CAD, coded as present if a participant reported any CAD. We assessed current and past smoking using validated questions from the Behavioral Risk Factor Surveillance System survey [9]. Participants were classified as ever-smokers versus never-smokers [10]. Exposure to secondhand smoke was not assessed. Missing data were not imputed.

We conducted two analyses. First, we examined the association of any CAD with smoking status, regardless of when the $\mathrm{CAD}$ was diagnosed. We used multivariable logistic regression to determine the association between smoking and CAD after adjustment for potential confounders including sex, race, age, education, income, health insurance status, marital status, region of residence and age at MS symptom onset (variable definitions listed below). We also adjusted for year of symptom onset in order to account for temporal changes in the diagnosis of MS and autoimmune disease. Statistical assumptions of the models were tested using standard methods.

Second, we restricted the analysis to participants who did not report any CAD at MS symptom onset $(n=8,349)$. Further excluding participants who were ex-smokers at MS symptom onset ( $n=990)$, we compared the risk of developing a CAD in current smokers versus never-smokers at MS onset. Each participant contributed person-years of follow-up from the year of symptom onset until the year of the first reported CAD, or the survey date, whichever came first. We estimated the incidence rate of CAD by dividing the number of participants reporting CAD diagnosed after MS onset by the number of person-years of follow-up.

Using a Cox proportional hazards model, we calculated the relative risk of a CAD associated with smoking. To account for changes in smoking status after MS onset, smoking status was included in the Cox model as a time-dependent covariate. Thus, if participants quit smoking before the onset of any autoimmune comorbidity, they were considered smokers only until they quit smoking. Covariates considered included sex, age at MS symptom onset and year of symptom onset. For independent variables, we tested the proportional hazards assumption using time-dependent covariates and graphical methods.

\section{Covariate Definitions}

Race was categorized as white (reference group) and other. Because of nonlinearity with respect to the dependent variable (CAD), current age was categorized into quintiles, with the lowest quintile as the reference group; year of symptom onset was categorized into <1990, 1990-1994, 1995-1999 and $\geq 2000$, and age at symptom onset was categorized as $\leq 25,<25-39$ and $\geq 40$ years. In the Cox model, both year of symptom onset and age at symptom onset were continuous variables. All statistical analyses used SAS version 9.1 (SAS Institute Inc., Cary, N.C., USA).

\section{Results}

As reported previously, 8,983 of the surveyed participants (56\%) responded to the questionnaire [6]. Seventyfive percent of the responders were women, $94 \%$ were white [6]. They had a mean (SD) age of 52.7 (10.4) years, a mean age at symptom onset of $31.2(9.0)$ years, a mean age at diagnosis of 38.2 (9.5) years, and a mean disease duration of 21.5 (11.2) years. Among the participants who reported their smoking status $(\mathrm{n}=8,929), 4,062(45.5 \%)$ were nonsmokers and 4,867 (54.5\%) were ever-smokers. As compared to ever-smokers, nonsmokers were more likely to be women, younger, of higher socioeconomic status and married, with a more recent year of symptom onset (table 1).

Of the 8,929 participants who reported their smoking status, 8,875 reported whether or not they had a CAD. In total, 1,649 participants $(18.5 \%)$ reported a CAD, and 410 (4.6\%) reported a preexisting autoimmune disease at MS symptom onset, while 781 (8.7\%) reported a preexisting autoimmune disease at diagnosis of MS (average time between onset and diagnosis: 7 years). Overall, thyroid disease was reported most frequently $(\mathrm{n}=871 ; 10 \%)$, followed by rheumatoid arthritis $(318 ; 3.6 \%)$, inflammatory bowel disease $(307 ; 3.5 \%)$, uveitis $(285 ; 3.3 \%)$, lupus (95; $1.1 \%)$ and Sjögren's syndrome (52; 0.6\%) [6]. These frequencies are similar to those reported in other North American MS populations (table 2). Among participants 
Table 1. Demographic characteristics of never-smokers and eversmokers

\begin{tabular}{|c|c|c|c|}
\hline & $\begin{array}{l}\text { Never- } \\
\text { smokers }\end{array}$ & $\begin{array}{l}\text { Ever- } \\
\text { smokers }\end{array}$ & $\mathrm{p}$ \\
\hline \multicolumn{4}{|l|}{ Sex, $n$} \\
\hline Female & $3,273(80.6)$ & $3,496(71.8)$ & $<0.0001$ \\
\hline Male & $789(19.4)$ & $1,371(28.2)$ & \\
\hline Age, years & $51.7 \pm 10.4$ & $53.5 \pm 10.4$ & $<0.0001$ \\
\hline \multicolumn{4}{|l|}{ Race, $\mathrm{n}$} \\
\hline White & $3,801(94.0)$ & $4,595(94.6)$ & 0.18 \\
\hline Nonwhite & $243(6.0)$ & $260(5.4)$ & \\
\hline \multicolumn{4}{|l|}{ Education, $\mathrm{n}$} \\
\hline$<$ High school & $37(0.92)$ & $124(2.6)$ & $<0.0001$ \\
\hline High school diploma & $1,126(27.9)$ & $1,976(40.9)$ & \\
\hline \multicolumn{4}{|l|}{ Associate's or } \\
\hline technical degree & 600 (14.9) & $839(17.4)$ & \\
\hline Bachelor's degree & $123(30.6)$ & $1,149(23.8)$ & \\
\hline Postgraduate degree & $1,039(25.7)$ & $746(15.4)$ & \\
\hline \multicolumn{4}{|l|}{ Income, $\mathrm{n}$} \\
\hline USD $<15,000$ & $331(9.9)$ & $549(13.4)$ & $<0.0001$ \\
\hline USD $15,000-30,000$ & $520(15.6)$ & $764(18.6)$ & \\
\hline USD $30,000-50,000$ & $638(19.1)$ & $959(23.3)$ & \\
\hline USD $50,000-100,000$ & $1,142(34.2)$ & $1,268(30.9)$ & \\
\hline USD $>100,000$ & $705(21.1)$ & $569(13.8)$ & \\
\hline \multicolumn{4}{|l|}{ Health insurance, $\mathrm{n}$} \\
\hline None & $38(1.0)$ & $87(1.8)$ & $<0.0001$ \\
\hline Public & $3,216(80.5)$ & $3,359(70.7)$ & \\
\hline Private & $738(18.5)$ & $1,304(27.5)$ & \\
\hline \multicolumn{4}{|l|}{ Marital status, $\mathrm{n}$} \\
\hline Single/divorced/widowed & $1,207(29.8)$ & $1,681(34.6)$ & $<0.0001$ \\
\hline Married/cohabiting & $2,847(70.2)$ & $3,175(65.4)$ & \\
\hline \multicolumn{4}{|l|}{ Region of residence, $\mathrm{n}$} \\
\hline West & $1,041(25.6)$ & $1,107(22.7)$ & 0.0002 \\
\hline Midwest & $1,024(25.2)$ & $1,296(26.6)$ & \\
\hline South & $1,090(26.8)$ & $1,234(25.4)$ & \\
\hline East & $907(22.3)$ & $1,230(25.3)$ & \\
\hline \multicolumn{4}{|l|}{ Year of symptom onset, $\mathrm{n}$} \\
\hline$<1990$ & $2,351(57.9)$ & $3,131(64.3)$ & $<0.0001$ \\
\hline 1990-1994 & $732(18.0)$ & $797(16.4)$ & \\
\hline 1995-1999 & $658(16.2)$ & $655(13.5)$ & \\
\hline$\geq 2000$ & $321(7.9)$ & $284(5.8)$ & \\
\hline Age at symptom onset, years & $31.1 \pm 9.0$ & $31.2 \pm 9.0$ & 0.65 \\
\hline
\end{tabular}

Values denote numbers with percentages in parentheses or means $\pm \mathrm{SD}$

reporting at least one of the specified autoimmune diseases, $183(12 \%)$ reported two, $32(2 \%)$ reported three, and $7(0.46 \%)$ reported four. After age adjustment, all autoimmune diseases except Sjögren's syndrome were more frequent than in the general population (table 2).

Among the 8,875 participants who reported their comorbidity and smoking status, 4,834 (54.5\%) ever smoked.
Among the ever-smokers, 951 (10.7\%) reported a CAD, while 693 never-smokers (7.8\%) reported a CAD ( $\mathrm{p}=$ 0.002). In a logistic model adjusting for age, sex, health insurance status and year of symptom onset, ever-smokers had increased odds of reporting a CAD (odds ratio: 1.22; 95\% CI: $1.08-1.38$ ).

Among the 7,830 participants without a CAD at MS onset who reported their smoking status and the age at which they started smoking, 3,035 (36.8\%) were current smokers, 990 (12.6\%) were ex-smokers, and 3,805 (48.6\%) were never-smokers. The frequency of later reporting a CAD varied with smoking status. Among never-smokers, $457(12.0 \%)$ developed a CAD, while $442(14.6 \%)$ of the ever-smokers developed one $(\mathrm{p}=0.002)$. The incidence rates for individual autoimmune comorbidities are shown in table 3. Smokers had increased risks of uveitis and rheumatoid arthritis.

In an unadjusted proportional hazards model, smokers had an increased risk of CAD (hazard ratio, HR: 1.23; 95\% CI: 1.08-1.41). After adjustment for sex, age at symptom onset and year of symptom onset, smokers still had the same risk of developing any CAD (HR: 1.23; 95\% CI: $1.08-1.41)$. We did a stratified analysis by sex. After adjustment, female smokers had a nearly $30 \%$ increased risk of developing a CAD (HR: 1.29; 95\% CI: 1.12-1.50). Male smokers did not have an increased risk (HR: 0.93 ; 95\% CI: $0.66-1.33$ ), but only 134 men developed a CAD, reducing the power of this analysis.

We evaluated the association of smoking with the risk of developing specific autoimmune diseases, although this was limited by small numbers. In the entire cohort, smoking was associated with a significantly increased risk of rheumatoid arthritis and uveitis (table 4). Although statistical significance was not reached for other conditions, the point estimates all exceeded 1. Among women, smoking was associated with an increased risk of rheumatoid arthritis. The number of men reporting autoimmune diseases was too small for further analysis.

\section{Sensitivity Analyses}

Sensitivity analyses were restricted to persons with (i) a relapsing course at onset (that is, excluding those with progressive disease at onset), and (ii) an age at symptom onset greater than 16 and less than 50 years (that is, typical ages at onset). Our findings were unchanged, with one exception (data not shown). In women, the association between smoking and inflammatory bowel disease became statistically significant (HR: 1.53; 95\% CI: $1.03-$ 2.28 ), and the point estimate was slightly higher than previously. 
Table 2. Percentages and 95\% CI of NARCOMS participants reporting autoimmune comorbidities after age adjustment to the 2000 US Census population, comparative results from North American MS populations ${ }^{1}$, and comparative results for the US general population where available

\begin{tabular}{|c|c|c|c|c|c|c|c|c|c|}
\hline \multirow[t]{3}{*}{ Comorbidity } & \multicolumn{4}{|c|}{ General population } & \multicolumn{3}{|c|}{ NARCOMS (age adjusted) } & \multicolumn{2}{|c|}{ MS populations } \\
\hline & ref. & men & women & both & men & women & both & ref. & $\%$ \\
\hline & No. & $\%$ & $\%$ & $\%$ & $\%$ & $\%$ & & No. & \\
\hline Rheumatoid arthritis & {$[22]$} & 0.66 & 1.31 & 1.05 & $3.1(1.9,4.3)$ & $3.0(2.5,3.4)$ & $3.0(2.6,3.4)$ & {$[7,12]$} & $2.0-3.0$ \\
\hline Sjögren's syndrome & {$[23]$} & 0.22 & 0.92 & 0.32 & $0.1(0.0,0.2)$ & $0.5(0.3,0.7)$ & $0.42(0.27,0.57)$ & {$[24]$} & 0 \\
\hline Systemic lupus erythematosus & {$[22]$} & 0.25 & 1.8 & 1.1 & $0.2(0.0,0.3)$ & $0.9(0.7,1.1)$ & $0.74(0.54,0.94)$ & {$[11,12]$} & $0.6-1.2$ \\
\hline Inflammatory bowel disease & {$[25]$} & 0.42 & 0.45 & 0.44 & $1.4(0.9,1.8)$ & $3.3(2.8,3.8)$ & $2.9(2.4,3.3)$ & {$[7,12]$} & $0.4-3.0$ \\
\hline Thyroid disease & {$[26]$} & & & 5.9 & $2.6(2.0,3.2)$ & $9.4(8.6,10.2)$ & $7.8(7.2,8.5)$ & {$[8,12]$} & 7.9-8.6 \\
\hline Uveitis & {$[27]$} & 0.095 & 0.13 & 0.12 & $2.3(2.0,2.7)$ & $2.3(2.0,2.7)$ & $2.4(2.0,2.8)$ & {$[28]$} & $26.0^{2}$ \\
\hline
\end{tabular}

Values in parentheses denote $95 \% \mathrm{CI}$.

${ }^{1}$ Findings from North American MS populations where studies included control groups, if available.

${ }^{2}$ Examination of 52 hospitalized patients with MS, not based on presentation with symptoms. Studies in France and Britain report uveitis frequencies of $1.1-2.4 \%[29,30]$.

Table 3. Incidence rates for individual CAD as well as rate ratios and $95 \% \mathrm{CI}$ in smokers and nonsmokers at MS onset $(\mathrm{n}=6,840)^{1}$

\begin{tabular}{|c|c|c|c|c|c|}
\hline \multirow[t]{2}{*}{ Comorbidity } & \multicolumn{3}{|c|}{ Incidence rate/100,000 } & \multirow[t]{2}{*}{$\mathrm{RR}$} & \multirow[t]{2}{*}{$95 \%$ CI } \\
\hline & overall & smokers & nonsmokers & & \\
\hline Thyroid & 346.7 & 352.3 & 366.9 & 0.92 & $0.77,1.10$ \\
\hline Rheumatoid arthritis & 128.5 & 152.7 & 114.2 & 1.43 & $1.07,1.92$ \\
\hline Uveitis & 115.7 & 133.9 & 107.0 & 1.38 & $1.02,1.88$ \\
\hline Inflammatory bowel disease & 102.2 & 120.1 & 93.8 & 1.24 & $0.89,1.73$ \\
\hline Systemic lupus erythematosus & 34.6 & 37.6 & 35.7 & 1.16 & $0.67,2.02$ \\
\hline Sjögren's syndrome & 22.1 & 25.9 & 20.6 & 1.43 & $0.73,2.99$ \\
\hline
\end{tabular}

$\mathrm{RR}=$ Rate ratio.

${ }^{1}$ Excludes participants who reported a CAD at MS onset, participants who did not report smoking status, age at starting smoking or age at quitting smoking.

\section{Discussion}

Case reports and population-based studies suggest that MS co-occurs with various autoimmune diseases [11]. Such observations triggered investigations of possible shared genetic susceptibility factors [7], but shared environmental factors could also explain the cooccurrence of disease. Several studies suggest that smoking is a risk factor for MS [3] and some autoimmune diseases [5]. Our findings suggest that smoking is an environmental risk factor for CAD in MS, particularly in women.
Our findings also suggest a possible explanation for disparate results regarding $\mathrm{MS}$ and $\mathrm{CAD}$ in previous large studies using different types of control groups [12, 13]. Using spousal controls, the Canadian Collaborative Project on Genetic Susceptibility to MS reported that persons with MS did not have an increased risk of CAD [12]. Another Canadian study used population-based controls and reported that the risk of inflammatory bowel disease was increased in MS [13]. If selected controls were less likely to smoke than cases with MS, an association between autoimmune disease and MS might be observed. If controls had a similar smoking frequen- 
Table 4. Adjusted HR and 95\% CI showing the association between smoking status (current vs. never) and development of CAD after onset of MS

\begin{tabular}{|c|c|c|c|c|}
\hline \multirow[t]{2}{*}{ Comorbidity } & \multicolumn{2}{|c|}{$\begin{array}{l}\text { Entire cohort }^{1} \\
(\mathrm{n}=6,840)^{3}\end{array}$} & \multicolumn{2}{|c|}{$\begin{array}{l}\text { Women }^{2} \\
(\mathrm{n}=5,176)^{3}\end{array}$} \\
\hline & HR & $95 \% \mathrm{CI}$ & HR & $95 \% \mathrm{CI}$ \\
\hline Any autoimmune comorbidity & 1.23 & $1.08,1.41$ & 1.29 & $1.12,1.50$ \\
\hline Thyroid & 1.12 & $0.93,1.36$ & 1.13 & $0.93,1.38$ \\
\hline Rheumatoid arthritis & 1.39 & $1.03,1.87$ & 1.64 & $1.16,2.31$ \\
\hline Uveitis & 1.38 & $1.01,1.89$ & 1.45 & $1.04,2.04$ \\
\hline Inflammatory bowel disease & 1.24 & $0.88,1.76$ & 1.42 & $0.96,2.08$ \\
\hline Systemic lupus erythematosus & 1.52 & $0.86,2.70$ & 1.40 & $0.77,2.56$ \\
\hline Sjögren's syndrome & 1.54 & $0.76,3.13$ & 1.56 & $0.74,3.26$ \\
\hline
\end{tabular}

${ }^{1}$ Adjusted for year of symptom onset, age at symptom onset and sex. ${ }^{2}$ Adjusted for year of symptom onset and age at symptom onset. ${ }^{3}$ Excludes participants who reported a CAD at MS onset, participants who did not report smoking status, age at starting smoking or age at quitting smoking.

cy to MS, as might be observed with spousal controls who often exhibit a high rate of smoking concordance and similarities in other important respects [14], no association would be observed. These scenarios assume that smoking is the only relevant factor, and this is unlikely to be the case; however, our findings suggest that future studies seeking to understand the relationships between CAD and MS should account for smoking status. This would be relevant for studies examining the frequency of CAD in MS, and for studies examining the influence of CAD on MS disease course, given the reported association of smoking with disease progression in MS [10].

Our response rate was slightly lower than the average response rate reported for published medical surveys [15], but nonresponse is unlikely to have biased the observed relationship within this cohort, unless smokers with autoimmune disease are more likely to respond than nonsmokers with autoimmune disease, contrary to usual patterns of nonresponse. Diagnoses of MS in this cohort are self-reported, but we confirmed diagnoses of MS in $98.7 \%$ of a random sample of this cohort [16], and studies in other MS populations also support the accuracy of self-reported diagnoses of MS [17]. Autoimmune conditions were self-reported, but studies support the accuracy of self-report for conditions which are chronic, disabling, or require ongoing care $[18,19]$. A large population-based study suggests that diagnoses of inflammatory bowel disease are accurately self-reported [18]. Further, work with our comorbidity questionnaire in other MS populations demonstrated substantial agreement $(\kappa=0.78 ; 0.66-0.89)$ between self-report and medical records for thyroid disease [20]. Although the sample sizes were too small to fully assess agreement for the other autoimmune diseases, reports of inflammatory bowel disease, rheumatoid arthritis and systemic lupus erythematosus were highly specific ( $>98 \%$ ) [17], and the frequency of these conditions in our cohort was similar to those in other North American MS populations (table 1). We did not capture family history of autoimmune disease, a potential surrogate for genetic factors associated with autoimmune disease. We lacked adequate data to examine a possible dose-response relationship between intensity of smoking and the risk of autoimmune disease, although duration of exposure was incorporated in our Cox proportional hazard models. Study strengths include the use of a large, well-characterized cohort of MS patients with a range of sociodemographic characteristics, experienced at completing questionnaires. We have shown previously that this cohort reports smoking status consistently over time, with a $\kappa=0.90$ (95\% CI: 0.890.92 ) for ever-smoking, and a $\kappa=0.92$ (95\% CI: $0.90-$ 0.94) for current smoking [21], and the questions we used have been widely used in national studies in the USA [9]. Results were consistent in the two analyses and robust to sensitivity analyses. By adjusting for year of symptom onset, we accounted for any temporal changes in the risk or diagnosis of CAD.

Within this large cohort of MS patients, smoking was associated with an increased risk of reporting a CAD af- 
ter MS onset. These findings should be replicated and extended in population-based cohorts, and suggest that investigators seeking to understand the relationships of comorbid disease to MS should jointly consider genetic and environmental factors, and that particular care should be given to selecting controls in observational studies. For clinicians, our findings also suggest that patients with MS should be counseled regarding the potential risks of developing CAD associated with smoking.

\section{Acknowledgments}

Supported partly by a Rudy Falk Clinician Scientist Award (to R.A.M.), the NIH, National Institute of Child Health and Human Development, Multidisciplinary Clinical Research Career Development Program Grant K12 HD04909, and the Consortium of Multiple Sclerosis Centers.

\section{Disclosure Statement}

The authors have no conflicts of interest to declare.

\section{References}

1 Munteis E, Cano JF, Flores JA, Martinez-Rodriguez JE, Miret M, Roquer J: Prevalence of autoimmune thyroid disorders in a Spanish multiple sclerosis cohort. Eur J Neurol 2007; 14:1048-1052.

-2 Brinar V, Petelin Z, Brinar M, Djakovic V, Zadro I, Vranjes D: CNS demyelination in autoimmune diseases. Clin Neurol Neurosurg 2006;108:318-326.

-3 Riise T, Nortvedt MW, Ascherio A: Smoking is a risk factor for multiple sclerosis. Neurology 2003;61:1122-1124.

$\checkmark 4$ Donaldson MJ, Pulido JS, Herman DC, Diehl N, Hodge D: Pars planitis: a 20-year study of incidence, clinical features, and outcomes. Am J Ophthalmol 2007;144:812-817.

$\checkmark 5$ Costenbader KH, Karlson EW: Cigarette smoking and autoimmune disease: what can we learn from epidemiology? Lupus 2006;15: 737-745.

-6 Marrie RA, Horwitz R, Cutter G, Tyry T, Campagnolo D, Vollmer T: Comorbidity, socioeconomic status, and multiple sclerosis. Mult Scler 2008;14:1091-1098.

7 Barcellos LF, Kamdar BB, Ramsay PP, DeLoa C, Lincoln RR, Caillier S, Schmidt S, Haines JL, Pericak-Vance MA, Oksenberg JR, Hauser SL: Clustering of autoimmune diseases in families with a high-risk for multiple sclerosis: a descriptive study. Lancet Neurol 2006; 5:924-931.

-8 Sloka JS, Pryse-Phillips WEM, Stefanelli M, Joyce C: Co-occurrence of autoimmune thyroid disease in a multiple sclerosis cohort. J Autoimmune Dis 2005;2:9.

9 Centers for Disease Control and Prevention: Behavioral Risk Factor Surveillance System Survey Questionnaire. Atlanta, US Department of Health and Human Services, 1995.

-10 Hernan MA, Jick SS, Logroscino G, Olek MJ, Ascherio A, Jick H: Cigarette smoking and the progression of multiple sclerosis. Brain 2005;128:1461-1465.

-11 Henderson RD, Bain CJ, Pender MP: The occurrence of autoimmune diseases in patients with multiple sclerosis and their families. J Clin Neurosci 2000;7:434-437.
12 Ramagopalan SV, Dyment DA, Valdar W, Herrera BM, Criscuoli M, Yee IM, Sadovnick AD, Ebers GC, Canadian Collaborative Study Group: The occurrence of autoimmune disease in Canadian families with multiple sclerosis: a population-based study. Lancet Neurol 2007;6:604-610.

13 Bernstein CN, Wajda A, Blanchard JF: The clustering of other chronic inflammatory diseases in inflammatory bowel disease: a population-based study. Gastroenterology 2005;129:827-836.

14 Kuo P-H, Wood P, Morley KI, Madden P, Martin NG, Heath AC: Cohort trends in prevalence and spousal concordance for smoking. Drug Alcohol Depend 2007;88: 122-129.

15 Asch DA, Jedrziewski MK, Christakis NA: Response rates to mail surveys published in medical journals. J Clin Epidemiol 1997;50: 1129-1136.

16 Marrie RA, Cutter G, Tyry T, Campagnolo D, Vollmer T: Validation of the NARCOMS Registry: diagnosis. Mult Scler 2007;13:770775.

17 Marrie RA, Yu N, Blanchard JF, Leung S, Elliott L: The rising prevalence and changing age distribution of multiple sclerosis in Manitoba. Neurology 2010;74:465-471.

18 Bernstein CN, Blanchard JF, Rawsthorne P. Wajda A: Epidemiology of Crohn's disease and ulcerative colitis in a central Canadian province: a population-based study. Am J Epidemiol 1999;149:916-924.

19 Okura Y, Urban LH, Mahoney DW, Jacobsen SJ, Rodeheffer RJ: Agreement between selfreport questionnaires and medical record data was substantial for diabetes, hypertension, myocardial infarction and stroke but not for heart failure. J Clin Epidemiol 2004; 57:1096-1103.

20 Horton M, Rudick RA, Hara-Cleaver C, Marrie RA: Validation of a self-report comorbidity questionnaire for multiple sclerosis. Neuroepidemiology 2010;35:83-90.

21 Marrie RA, Cutter G, Tyry T, Campagnolo D, Vollmer T: Smoking status over two years in patients with multiple sclerosis. Neuroepidemiology 2009;32:72-79.
22 Lawrence RC, Helmick CG, Arnett FC, Deyo RA, Felson DT, Giannini EH, Heyse SP, Hirsch R, Hochberg MC, Hunder GG, Liang MH, Pillemer SR, Steen VD, Wolfe F: Estimates of the prevalence of arthritis and selected musculoskeletal disorders in the United States. Arthritis Rheum 1998;41:778-799.

23 Helmick CG, Felson DT, Lawrence RC, Gabriel S, Hirsch R, Kwoh CK, Liang MH, Kremers HM, Mayes MD, Merkel PA, Pillemer SR, Reveille JD, Stone JH, National Arthritis Data Workgroup: Estimates of the prevalence of arthritis and other rheumatic conditions in the United States. Part I. Arthritis Rheum 2008;58:15-25

24 Noseworthy JH, Bass BH, Vandervoort MK, Ebers GC, Rice GP, Weinshenker BG, McLay CJ, Bell DA: The prevalence of primary Sjögren's syndrome in a multiple sclerosis population. Ann Neurol 1989;25:95-98.

-25 Kappelman MD, Rifas-Shiman SL, Kleinman K, Ollendorf DA, Bousvaros A, Grand RJ, Finkelstein JA: The prevalence and geographic distribution of Crohn's disease and ulcerative colitis in the United States. Clin Gastroenterol Hepatol 2007;5:1424-1429.

26 Hollowell JG, Staehling NW, Flanders WD, Hannon WH, Gunter EW, Spencer CA, Braverman LE: Serum TSH, $\mathrm{T}_{4}$, and thyroid antibodies in the United States population (1988 to 1994): National Health and Nutrition Examination Survey (NHANES III). J Clin Endocrinol Metab 2002;87:489-499.

27 Gritz DC, Wong IG: Incidence and prevalence of uveitis in Northern California. Ophthalmology 2004;111:491-500.

28 Breger BC, Leopold IH: The incidence of uveitis in multiple sclerosis. Am J Ophthalmol 1966;62:540-545.

29 Edwards LJ, Constantinescu CS: A prospective study of conditions associated with multiple sclerosis in a cohort of 658 consecutive outpatients attending a multiple sclerosis clinic. Mult Scler 2004;10:575-581.

-30 Biousse V, Trichet C, Bloch-Michel E, Roullet E: Multiple sclerosis associated with uveitis in two large clinic-based series. Neurology 1999;52:179-181 